\title{
$O$-glycosylation in plant and mammal cells: the use of chemical inhibitors to understand the biosynthesis and function of $O$-glycosylated proteins
}

\author{
Ludovic Mewono ${ }^{3}$, Eric Nguema-Ona², Maxime Gotté ${ }^{2}$, Abdul Salam Koroney², Marie-Laure Follet \\ Gueye $^{2}$, Azeddine Driouich², Maïté Vicré-Gibouin ${ }^{2}$, Sophie Aboughe-Angone ${ }^{1 ⿴}$
}

\begin{abstract}
Glycosylation is the most common posttranslational modification of proteins and consists of the addition of sugar moiety to proteins. The resulting glycosylated proteins are often secreted to the extracellular compartment or integrated into different cell organelles. This modification was identified in plant as well as in mammalian cells. A number of plant and mammal proteins are either $N$ - or $O$-glycosylated. This review focuses on $O$-glycosylation which refers to linkage of a glycan to hydroxyl group of serine, threonine or proline residues. $O$-glycosylation can be altered by the action of chemical inhibitors. For instance, 3,4-dehydro-L-proline, ethyl 3,4-dehydroxy benzoate and $\alpha, \alpha$-dipyridyl inhibit the activity of prolyl4-hydroxylase, a key enzyme
\end{abstract}

Received: 31 May 2014

Accepted revised version: 18 July 2014

Published online: 01 April 2015

(C) Mewono et al. (2015)

Publisher: Horizon e-Publishing Group

\section{CITATION}

Mewono, L., E. N. Maxime Gotté, A. S. Koroney, M. F. Gueye, A. Driouich, M. Vicré-Gibouin, and S. Aboughe-Angone. 2015. O-glycosylation in plant and mammal cells: the use of chemical inhibitors to understand the biosynthesis and function of O-glycosylated proteins. Plant Science Today 2(2): 43-51. doi: 10.14719/pst.2015.2.2.67

AUTHORS' AFFILIATION

1 Institut de Pharmacopée et de Médecine Traditionnelle CENAREST, BP: 5011 Libreville (Gabon)

2 Laboratoire de Glycobiologie et Matrice Extracellulaire Végétale, EA4358, GRR VASI PRES Normandie Université, Université de Rouen, 76821 Mont-Saint-Aignan, France

3 Groupe de recherche en immunologie et microbiologie appliquée (GRIMA), Département des Sciences de la Vie et de la Terre- Ecole Normale Supérieure, BP: 17009 Libreville

CORRESPONDENCE

$\triangle$ Sophie Aboughe Angone Email: sophie.aboughe@gmail.com for plant $O$-glycosylation. In addition, a small molecule inhibitor designated 1-68A inhibits the polypeptide $\mathrm{N}$-acetylgalactosaminyltransferases of mammalian cells. The aim of this review is to summarize the role and mechanism of action of these inhibitors of $\boldsymbol{O}$-glycosylation and their impact on cell development in plants and mammals.

Keywords: O-glycosylation; 3,4-dehydro-L-proline; 3,4-dehydroxybenzoate; prolyl-hydroxylase; $\mathrm{N}$-acetyl-galactosaminyltransferases; $\alpha, \alpha$-dipyridyl

\section{Abbreviations:}

PTM: posttranslational modification

ER: endoplasmic reticulum

HRGPs: hydroxyproline-rich glycoproteins

AGPs: arabinogalactan-proteins

Hyp: hydroxyproline

P4-Hs: prolyl-4-hydroxylases

Ser/Thr: serine/threonine

GalNAc-Ts: N-acetylgalactosaminyltransferases

3,4-DHP: 3,4-dehydro-L-proline

3,4-EDHB: ethyl-3,4-dehydroxy benzoate

DP: $\alpha, \alpha$-dipyridyl

GalNAc-O-bn: benzyl-N-acetyl-a-galactosamide

ppGNTases: polypeptide $\mathrm{N}$-acetylgalactosaminyltransferases

\section{Introduction}

Protein glycosylation is one of the major posttranslational modification (PTM) occurring in eukaryotic cells (from unicellular to multicellular organisms). This PTM which generally follows/ accompanies the synthesis of polypeptides in the endoplasmic reticulum (ER) is initiated in this same compartment, and completed in the Golgi apparatus. Glycosylated proteins follow the secretory pathways and are either released in the extracellular compartments (plasma membrane, cell wall, cell surface matrix) or destined to different organelles within the cell (e.g., lysosomes, vacuoles). Both $N$-linked and $O$-linked glycosylation processes have been found and 
studied in plant and animal cells. $N$-glycosylation is very well described and has been the object of many studies, reviews and biotechnological applications (Strasser, 2009; Bardor et al., 2010; Gomord et al., 2010), whereas $O$-glycosylation is less studied in general, and is the focus of this paper. $O$-glycosylated proteins are known to play important functions in animal and plant cells including cell-cell interactions, signaling, recognition, and scaffolding. Protein glycosylation is also required for maintaining the folding, the stability and the activity of (summarized in Fig. 1).

In plants, $O$-glycosylated proteins have been abundantly reported within a family of secreted cell wall proteins, the hydroxyproline-rich glycoproteins (HRGPs). HRGPs constitute a superfamily composed of arabinogalactan-proteins (AGPs), extensins, and proline-rich proteins (Showalter et al., 2010; Xu et al., 2008; Velasquez et al., 2011). HRGPs are variably glycosylated (Showalter, 1993). These proteins undergo an essential modification namely the conversion of

\section{Plant}

Ara-Ara- Gal $\beta 1-6 \mathrm{Gal} \beta 1$

${ }_{\ldots . \mathrm{Gal} \beta 1-3 \mathrm{Gal} \beta 1-3 \mathrm{Gal} \beta 1}^{\ldots . \mathrm{Gal} \beta 1}{ }_{6}^{6} \mathrm{Gal} \beta 1-O-\mathrm{Hyp}$

Type II arabinogalactan

Arao 1-3Araß1-2 Araß1-2 Araß1-O-Hyp

Extensin type $O$-glycan

Gal 1 -3Gal 1 1-O-Ser

Solanaceous lectin-type $O$-glycan

Animal

.....GLcA $\beta 1-3 \mathrm{Gal} \beta 1-3 \mathrm{Gal} \beta 1-4 \mathrm{Xyl} \beta 1-O$-Ser

GlcNAc $\beta 1-3$ Fuc $\beta 1$ - $O$-Ser

Gal $\beta 1$-3 GalNAc $\alpha 1-O$-Ser/Thr

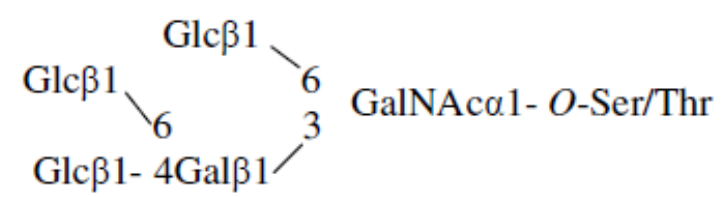

3

Glc $\beta 1$

2-O-Me Fuc $\alpha$ 1-2 Gal $\beta 1-3$ GalNAc $\alpha 1-O$-Ser/Thr

GlcNAc $\beta 1-6(G a l \beta 1-3)$ GalNAc $\alpha-S e r / T h r$

GlcNAc $\beta 1 \rightarrow 3$ GalNAc $\alpha-$ Ser/Thr

GlcNAc $\beta 1 \rightarrow 3($ GlcNAc $\beta 1 \rightarrow 6)$ GalNAc $\alpha$ Ser/Thr

\section{Glycosaminoglycan (core region)}

$O$-linked fucose

Mucin-type core 1 glycan (fly)

Mucin-type (worm, example)

\author{
Mucin-type core 1 glycan (Toxocara) \\ Mucin-type core 2 glycan \\ Mucin-type core 3 glycan \\ Mucin-type core 4 glycan
}

Fig. 1. 0-linked glycans in the process of general 0-glycosylation in the plant and mammalian cells (Wilson Iain, 2002; Kirk Bergstrom and Xia, 2013)

secreted proteins (Apweiler et al., 1999; Haltiwanger and Lowe, 2004). The structure of $O$-linked glycans of plant versus animal glycoproteins is quite different proline residues into hydroxyproline (Hyp) by a multigene family of enzymes, the prolyl-4-hydroxylases (P4-Hs) (Vlad et al., 2007) that act within the 
endoplasmic reticulum and the Golgi apparatus. The Hyp residues are then glycosylated either with arabinogalactan chains, with short arabinan chains, or with single galactose residues by different glycosyltransferases in the Golgi apparatus (Shpak et al, 1999; Showalter, 2001; Wilson Iain, 2002; Velasquez et al., 2011; Velasquez et al., 2012). Molecular genetics approaches coupled to analytical techniques have shown that glycosylation of Hyp residues is dependent on a variety of consensus motifs widespread within the peptide amino acid sequence. $O$-glycosylation leading to arabinosylation occurs on clustered Ser-(Hyp)4 contiguous sequences (contigs), whereas $O$-glycosylation leading to arabinogalactosylation occurs on Hyp-alanine, Hyp-serine, Hyp-threonine and Hyp-valine contigs (Kieliszewski and Shpak, 2001; Shpak et al, 2001; Tan et al., 2003; Tan et al., 2004; Estévez et al., 2006; Xu et al., 2008).

In mammals, $O$-glycosylation is not predominantly found on proline residues; it does also occur on serine/threonine (Ser/Thr), tyrosine and hydroxylysine amino acids. The most common of these modifications is the mucin-type $O$-glycosylation, which is widespread in the animal kingdom (Bennett et al., 2012). In mammals, initiation of mucin-type $O$-glycosylation can be performed by up to 20 different UDP-GalNac polypeptide $\mathrm{N}$-acetylgalactosaminyltransferases (GalNAc-Ts) which catalyze the transfer of a single $\mathrm{N}$-acetylgalactosamine (GalNAc) residue to serine/threonine amino acids (GalNAca Ser/Thr). Further elongation of GalNAc residues is performed by Golgi-located glycosyltransferases and results in different $O$-glycan structures including core 1 to core 4 structures. Mucin-type $O$-linked glycoproteins are involved in many biological processes such as the regulation of serum half-life of cytokine, the alteration of glycans on cancer cells and the lubrication of variety of intestinal tissues. Mucins play structural role and contribute to mechanical properties, organization, and shape of tissues, and interact with cells via different receptors. They also regulate their migration, proliferation, and differentiation (Ricard-Blum, 2010). $O$-glycosylation has also been well studied on collagen, which is the most abundant proteins in mammals ( $\sim 30 \%$ of total protein mass in certain cells). Collagen contains more than $10 \%$ of proline residues. It does also contain lysine residues. The enzymes which hydroxylate these amino acids are the prolylhydroxylase and lysylhydroxylase (Ricard-Blum, 2010; Rosenbloom and Prockop, 1970).

Understanding $O$-protein glycosylation has direct implications in basic research but also in several areas of biotechnology, including molecular pharming, or novel strategies of plant/crop protection. Progress in such an understanding has been facilitated by different approaches including molecular genetics and pharmacological approaches. Here, we have chosen to present some drugs/inhibitors which have been used to unravel plant and mammal $O$-glycosylation. Prospects in application, especially in plant biotechnology, are also presented.

\section{Inhibiting $\boldsymbol{O}$-glycosylation in plants using chemical inhibitors}

$\mathrm{P} 4$-Hs perform a key step in plant $O$-glycosylation, which is the hydroxylation of proline residues. Direct or indirect inhibition of the action of this enzyme has been used to study the function of $O$-glycosylation in plants. To interfere with the process of $O$-glycosylation, various substances have been employed including 3,4-dehydro-L-proline (3,4-DHP), ethyl-3,4-dehydroxy benzoate (3,4-EDHB) and $\alpha, \alpha$-dipyridyl.

3,4-DHP is an analog of proline which can be incorporated into the polypeptide chain, and rapidly and irreversibly inhibits peptidyl proline hydroxylation. 3,4-DHP has been shown to inhibit P4-Hs action in plant cells when applied at micromolar concentrations (Cooper and Varner, 1983). The authors have shown that treatment of discs of phloem parenchyma tissue from roots of Daucus carota with $5 \mu \mathrm{M}$ and $50 \mu \mathrm{M} 3,4-\mathrm{DHP}$ inhibits the hydroxylation of proline by $70 \%$ and $90 \%$, respectively. An unpublished study has shown that 3,4-DHP-treated tobacco BY-2 cells were able to alter the molecular weight as well as the carbohydrate composition of secreted AGPs (Aboughe-Angone Sophie, Nguema-Ona Eric, Azeddine Driouich, article in preparation). 3,4-DHP treatment of soybean cells was also shown to induce disappearance of the major PRPs from the cell wall of cultured cells (Schmidt et al., 1991), and 3,4-DHP-treated protoplasts do not develop osmotic stability and do initiate mitosis. 3,4-DHP-treated carrot root cells continue to synthesize and secrete structurally abnormal extensin HRGPs that might be expected to function abnormally (Cooper et al., 1994). Others studies showed that treatment of onion root cells by 3, 4-DHP resulted on a $56 \%$ decrease in hydroxyproline content (Tullio et al., 1999). Recently, $\mathrm{Xu}$ et al. (2011), have shown that 3,4-DHP inhibited the proliferation of embryogenic cells of banana and decreased the rate of embryo germination. In the context of plant microbe interactions, 3,4-DHP has been used to investigate the role of plant AGPs in root colonization by soil rhizobacteria (Vicré et al., 2005). It has been shown that 3,4-DHP induced alteration of AGP glycosylation and altered rhizobacteria ability to colonize Arabidopsis roots (Nguema-Ona et al., 2013). Altogether, these studies showed that inhibition/alteration of plant 
$O$-glycosylation by 3,4-DHP treatment affects many aspects of plant development and interaction with microbes. Interestingly, in mammalian cells, proline hydroxylation in collagen is also inhibited by 3,4-DHP treatment (see below).

$O$-glycosylation in the plant cells can also be inhibited by ethyl-3,4-dehydroxy benzoate (3-4-EDHB) and $\alpha, \alpha$-dipyridyl (DP) (Velasquez et al, 2011). 3,4-EDHB binds to the active site of prolyl4-hydroxylase, while $\alpha, \alpha$-dipyridyl (DP) chelates a co-factor required for P4-Hs activity. Velazquez et al. have shown that transgenic Arabidopsis roots expressing the fusion LeAGP1::GFP treated at nanomolar range (48 to $219 \mathrm{nM}$ ) with these two drugs exhibited $50 \%$ growth inhibition of their root hairs. These treatments also caused the accumulation of non-glycosylated $\sim 42 \mathrm{KD}$ forms of LeAGP1-GFP and fully $O$-glycosylated $\sim 150$ to $200 \mathrm{KD}$ form. The authors also linked the absence of $O$-glycosylation of extensins in the p4-h Arabidopsis mutants to the abnormal root hair phenotype observed.

Altogether, these studies show that the use of $O$-glycosylation inhibitors in plants affect considerably various aspects of growth and survival supporting the importance of the $O$-glycosylation in the function of plant cells.

Inhibiting $O$-glycosylation in mammals using chemical inhibitors

In mammals, proline, but also serine, threonine, and tyrosine amino acids can be $O$-glycosylated. Different enzymes perform the addition of glycosidic residues on
$O$-glycosylation. Collagens and mucins are well studied mammal-O-glycosylated proteins. Interestingly, collagen $O$-glycosylation often occurs on Hyp residues while mucin $O$-glycosylation occurs on serine or threonine residues.

Rosenbloom and Prockop (1970) have studied the effect of 3,4-DHP treatment on collagen $O$-glycosylation. They have found that 3,4-DHP decreased the hydroxylation of proline and lysine residues in collagen and that the altered collagen could not be extruded from cartilage cells as rapidly as that of control samples. The effects observed following 3,4-DHP treatment were comparable to those observed with azetidine-2-carboxylic acid and cis-4-fluoroproline (analog of proline) but were slightly less pronounced (Takeuchi and Prockop, 1969, Takeuchi et al., 1969). In the study on collagen synthesis and prolyl hydroxylase activity in mammalian cell cultures, Kerwar and Felix (1976) showed that, in addition to decreasing the hydroxylation of proline residues and the secretion of collagen from cells, 3,4-DHP also inhibited the incorporation of glycine and lysine into collagen. 3,4-DHP also prevented excessive collagen deposition during wound healing and inhibited fibrosis development (Kerwar and Felix, 1976).

By contrast with collagen-type $O$-glycosylation, mucin-type $O$-glycosylation occurs on serine and threonine residues, and occurs also on many other glycoproteins (Tarp and Clausen, 2008). This type of $O$-glycosylation constitutes the most common type of $O$-linked glycosylation found in mammals and other eukaryotes (Hang and Bertozzi, 2005). Here,

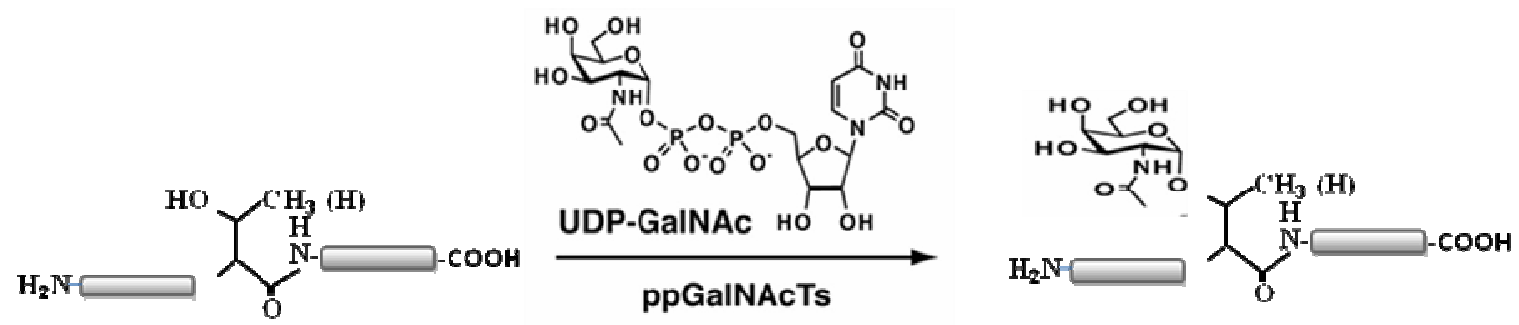

Protein substrate

Mucin-type O-linked glycoprotein

Fig. 2. Initiation of mucin-type $O$-linked glycosylation biosynthesis by the ppGalNAc-Ts which utilize UDP-GalNAc as the nucleotide sugar donor and modify protein substrates (Hang and Bertozzi, 2005; Tian et al., 2004)

the polypeptidic backbones. Therefore, different drugs targeting different key enzymes have also been identified and used to study mammal-specific glycosylation is initiated by the ppGNTases. These are enzymes that initiate mucin-type $O$-linked glycosylation by transfer of GalNAc from the nucleotide donor 
UDP-GalNAc to Ser/Thr residues of a polypeptide substrate, as shown in Fig. 2 (Tian et al., 2004). This enzyme plays a pivotal role in cell-cell communication and tissue protection (Hang et al., 2004). They have identified a small molecule (1-68A) from a uridine-based library, which inhibits $\mathrm{N}$-acetylgalactosaminyltransferases in mammalian cells and consequently disrupts $O$-glycosylation. To define the
O-glycans increase HRGPs solubility and thermal stability as well as resistance to proteolytic degradation (Ferris et al, 2001; Kieliszewski et al, 2010; Lamport et al., 2011). In recent studies, it has been shown that $O$-glycosylation of hydroxyproline residues increase the thermal stability of the prolyproline type II helix and the presence of $\beta$-O-glycosylated proteins indicated an increase in conformational stability (Owens et al., 2010;

Table 1: Possible functions in plant and mammalian cells

\begin{tabular}{|l|l|}
\hline Role of $\boldsymbol{O}$-glycosylation in Plant cell walls & Role of $\boldsymbol{O}$-glycosylation in mammalian cells \\
\hline- protein stability & - protein stability \\
- somatic embryogenesis & - heat resistance \\
- - cell division and cell expansion & - protein expression and processing \\
- reproductive development & - protease resistance \\
- abiotic stress responses & - cellular interactions \\
$-\quad$ xylem differentiation & - hydrophilicity and antigenicity \\
$-\quad$ hormone signaling pathways & - modulation of enzymes activity \\
\hline
\end{tabular}

biological roles of the GalNAc-Ts, Tian et al. (2004) subjected NIH3T3 mouse fibroblast cells to 1-68A. The authors observed a marked decrease of protein glycosylation and induction of cell apoptosis. Over-expression of certain isoforms of GalNAc-Ts restored cell surface glycosylation and rescued the inhibitor-induced apoptosis suggesting that the enzymes and $O$-glycosylation have an important role in the regulation of apoptosis. Also, inhibition of $O$-glycosylation seems to play a key role in the growth of cancer cells (Patsos et al, 2005). For instance, benzyl-N-acetyl-a-galactosamide (GalNAc-O-bn) inhibits the $O$-gylcosylation of KL- 6 mucin which is implicated in the development of aggressive metastasis in human pancreatic carcinoma (Xu et al., 2008).

\section{Comparative functional aspects of plant and mammal $\boldsymbol{O}$-glycosylated proteins}

Plant and mammalian $O$-glycans are usually considered as structurally different, but the studies of $O$-glycosylation inhibitors in the mammalian and plant cells show that both plant and animal $O$-glycosylations are involved in the stability and the conformation of glycoproteins. In the case of extensins, $O$-glycosylation appears to stabilize the helical conformation whereas incomplete hydroxylation of $O$-glycosylation would promote a flexible and disorganized conformation (Velasquez et al., 2011). It has been suggested that
Velasquez et al, 2011). Interestingly plant $O$-glycosylated proteins are also suggested to play active role in plant defense mechanisms and signaling (Nguema-Ona et al., 2013). PTMs of $O$-glycosylated cell wall proteins are important to cell differentiation and plant development. $O$-glycosylated proteins like arabinogalactan-proteins and extensins are involved in the regulation of plant growth, somatic embryogenesis, and development processes, cell division and cell expansion, reproductive development, biotic and abiotic stress responses, xylem differentiation, and hormone signaling pathways (Seifert and Roberts, 2007; Zhang et al., 2008; Ellis et al., 2010; Velasquez et al, 2011; Xu et al., 2011; Velasquez et al., 2012). The slightest inhibition or the disruption of this PTM affects the process in which these molecules are involved in plant development. However, $O$-glycosylation type defines the glycomodules responsible for their shape, size, stability, and biological functions in HRGPs.

These functions are similar in the mammalian cells. Several examples can illustrate the role of $O$-glycosylation in mammalian cells. In general, $O$-linked glycans maintain protein stability, heat resistance, antigenicity, cellular interactions, hydrophilicity, and modulation of enzymatic activity and protease resistance by steric hindrance, protein expression and processing (Van den Steen et al., 1998; Wopereis et al, 2006). $O$-glycosylation would induce the extended and 
rigid conformation in the mucin-1 which is a polymorphic, highly glycosylated, type I transmembrane glycoprotein and this allows this molecule to interfere with cell-cell and cell-matrix interactions (Tarp and Clausen, 2008). Another important function of $O$-linked sugars is to mediate recognition between proteins. $O$-glycosylation is also essential for the expression or biological activity of many proteins. For example,
Wiskott-Aldrich syndrome and cancer (Tsuboi and Fukuda, 2001; Berger, 1999; Brockhausen, 1999). $O$-linked glycans are also known to have an effect on immunologic recognition, for example, the $\mathrm{ABO}$ blood group antigens and recognition by antibodies (van den Steen et al, 2000). In addition, $O$-glycosidic structures play an important role in the localization and traffic of proteins, and make them more resistant to proteases,

Table 2: Chemical structures of inhibitors that are commonly used to inhibit plants or mammalians $O$-glycosylation

\begin{tabular}{|c|c|}
\hline & NAMES \\
\hline
\end{tabular}

$O$-glycans influence the expression of glycophorin A at the cell surface of human erythrocytes (Remaley et al, 1991), or the biological activity of interleukin-5 (Kodama et al, 1993) and human lactase phlorizin hydrolase (Naim and Lentze, 1992). Altered glycosylation directly leads to development of certain diseases such as hematological disorders, increase their stability, avoid or reduce the effects related to oxidation, denaturation by $\mathrm{pH}$, precipitation or aggregation. A specific glycan may be involved in several functions in different places and different times (Varki, 1993). The different possible functions of O-glycosylation are summarized in Table 1.

In Table 2, we summarize also the structure of 
chemical inhibitors commonly used to inhibit plant or mammalian $O$-glycosylation.

\section{General Conclusions}

Protein glycosylation in general and $O$-glycosylation in particular, is a fundamental process for the function plant and mammalian cells. $O$-glycosylation alteration in directly impacts cell development and survival of living organisms. In plants, such an alteration affects plant development. Moreover $O$-glycosylated plant cell wall proteins play an important role in plant survival. In mammals, $O$-glycosylation accompanies many secreted proteins that have important roles in signaling and communication. Proper $O$-glycosylation is also often directly responsible for the biological activities of these proteins. The use of chemical inhibitors, in combination with molecular genetics approaches and analytical chemistry, has largely contributed to the study of the structure and function of $O$-glycosylated proteins.

Understanding and harnessing $O$-glycosylation has implications in production of $O$-glycosylated biopharmaceuticals for mammal therapy. Given their natural ability to perform $O$-glycosylation, plants are suitable candidate for the production of therapeutic glycoproteins requiring $O$-glycans (Gomord et al, 2010; Sola and Griebenow, 2010; Strasser, 2013). A recent study has described $O$-glycosylation of a recombinant human IgA1 expressed in transgenic maize (Karnoup et al., 2005). Addition of small arabinan chains (via an addition of Hyp residues to the polypeptide sequence) to therapeutic polypeptides expressed in plants was also shown to increase the half-life of the recombinant molecule and its activity.

Plant $O$-glycosylated proteins such as AGPs have also emerged as interesting players in plant defense against soil pathogens (Nguema-Ona et al., 2013). Thus, future applied research in plants should evaluate the possibility of massive production of $\mathrm{O}$-glycosylated proteins of interest.

\section{Competing interests}

The authors declare that they have no competing interests.

\section{Acknowledgments}

We would like to thank present and former colleagues who contributed to the work described in this review and for their comments on the manuscript.

\section{References}

Apweiler, R., H. Hermjakob, and N. Sharon. 1999. On the frequency of protein glycosylation, as deduced from analysis of the SWISS-PROT database. Biochemica et
Biophysica Acta 1473: 4-8. doi: 10.1016/S0304-4165 (99) 00165-8.

Bardor, M., J.A. Cremata, and P. Lerouge. 2010. Glycan Engineering in Transgenic Plants. Annual Plant Reviews: Plant Polysaccharides, Biosynthesis and Bioengineering 41: 409 - 424. doi: 10.1002/9781444391015.ch17.

Bennett, E.P., U. Mandel, H. Clausen, T.A. Gerken, T.A. Fritz, and L.A. Tabak. 2012. Control of mucin-type O-glycosylation: A classification of the polypeptide GalNAc-transferase gene family. Glycobiology 22: 736-756. doi: 10.1093/glycob/cwr182. Epub 2011 Dec 18.

Berger, E.G. 1999. Tn-syndrome. Biochemica et Biophysica Acta 1455: 255-268. doi: 10.1016/S0925-4439 (99) 00069-1.

Brockhausen, I. 1999. Pathways of 0-glycan biosynthesis in cancer cells. Biochemica et Biophysica Acta 1473: 67-95. doi: 10.1016/S0304-4165 (99) 00170-1.

Cooper, J.B, and J.E. Varner. 1983. Selective inhibition of proline hydroxylation by 3,4-dehydroproline. Plant Physiology 73: 324-328. doi: 10.1104/pp.73.2.324.

Cooper, J.B., J.E. Heuser, and J.E. Varner. 1994. 3,4-Dehydroproline inhibits cell wall assembly and cell division in tobacco protoplasts. Plant Physiology 104: 747-752. doi: 10.1104/pp.104.2.747.

Ellis, M., J. Egelund., C. Schultz, and A. Bacic. 2010. Arabinogalactan-proteins (AGPs): key regulators at the cell surface? Plant Physiology 153: 403-419. doi: 10. 1104/pp.110.156000.

Estévez, J., M.J. Kieliszewski., N. Khitrov, and C. Somerville. 2006. Characterization of synthetic hydroxyproline-rich proteoglycans with arabinogalactan-protein and extensin motifs in Arabidopsis. Plant Physiology 142: 458-470. doi: $10.1104 /$ pp.106.084244.

Ferris, P.J., J.P. Woessner., S. Waffenschmidt., S. Kilz., J. Drees, and U.W. Goodenough. 2001. Glycosylated polyproline II rods with kinks as a structural motif in plant hydroxyproline-rich glycoproteins. Biochemistry 40: 2978-2987. doi: 10.1021/bi0023605.

Gomord, V., A.C. Fitchette., L. Menu-Bouaouiche., C. Saint-Jore-Dupas., C. Plasson., D. Michaud, and L. Faye. 2010. Plant-specific glycosylation patterns in the context of therapeutic protein production. Plant Biotechnology Journal 8: 564-587. doi: 10.1111/ j.1467-7652.2009.00497.x.

Haltiwanger, R.S, and J.B Lowe. 2004. Role of Glycosylation in Development. Annual Review of Biochemistry. 73: 491-537. doi: 10.1146/annurev.biochem.73.011303.074043

Hang, H.C, and C.R. Bertozzi. 2005. The chemistry and biology of mucin-type O-linked glycosylation. Bioorganic \& Medicinal Chemistry. 13: 5021-5034. doi: 10.1016/j.bmc.2005.04.085.

Hang, H.C., C. Yu., K.G. Ten Hagen., E. Tian., K.A. Winans., L.A. Tabak, and C.R. Betozzi. 2004. Small Molecule Inhibitors of Mucin-Type $O$-Linked Glycosylation from a Uridine-Based Library. Chemistry Biology. 11: 337-345. doi: 10.1016/j.chembiol.2004.02.023. 
Karnoup, A.S., V. Turkelson, and W.H.K. Anderson. 2005. 0-Linked glycosylation in maize-expressed human IgA1. Glycobiology. 15: 965-981. doi: 10.1093/GLYCOB/CWI077.

Kerwar, S.S, and A.M. Felix. 1976. Effect of L-3,4-Dehydroproline on Collagen Synthesis and Hydroxylase Activity in Mammalian Cell Cultures Proly. The Journal of Biological Chemistry. 251: 503-509. PMID: 173719.

Kieliszewski, M.J, and E. Shpak. 2001. Synthetic genes for the elucidation of glycosylation codes for arabinogalactan-proteins and other hydroxyproline-rich glycoproteins. Cellular and Molecular Life Sciences. 58: 1386-1398. PMID: 11693521.

Kieliszewski, M.J., D.T.A. Lamport., L. Tan, and M.C. Cannon. 2010. Plant Polysaccharides, Biosynthesis and Bioengineering. In: Annual Plant Reviews. P. Ulvskov, Ed . p. 321-342. doi: 10.1002/9781444391015.

Kirk Bergstrom, S.B, and L. Xia. 2013. Mucin-type O-glycans and their roles in intestinal homeostasis. Glycobiology. 0: 1-12. doi: 10.1093/glycob/cwt045.

Kodama, Y., C.J. Boreiko., S.C. Maness, and T.W. Hesterberg. 1993. Cytotoxic and cytogenetic effects of asbestos on human bronchial epithelial cel1s in culture. Carcinogenesis. 14: 691-697. doi: 10.1093/carcin/14.4.691.

Lamport, D.T.A., M.J. Kieliszewski., Y. Chen, and M.C. Cannon. 2011. Role of the Extensin Superfamily in Primary Cell Wall Architecture. Plant Physiology. 156: 11-19. doi: 10. 1104/pp.110.169011.

Naim, H.Y, and M.J. Lentze. 1992. Impact of 0-glycosylation on the function of human intestinal lactase-phlorizin hydrolase.Characterization of glycoforms varying in enzyme activity and localization of 0 -glycoside addition. The Journal of Biological Chemistry. 267: 25494-504.

Nguema-Ona, E., M. Vicré-Gibouin., M.A. Cannesan, and A. Driouich. 2013. Arabinogalactan proteins in root-microbe interactions. Trends in Plant Science. 18: 440-449.doi: http://dx.doi.org/10.1016/j.tplants.2013.03. 006.

Owens, N.W., J. Stetefeld, E. Lattová, and F. Schweizer. 2010. Contiguous $O$-galactosylation of $4(\mathrm{R})$-hydroxy-l-proline residues forms very stable polyproline II helices. Journal of the American Chemical Society. 132: 5036-5042. doi: $10.1021 / \mathrm{ja} 905724 \mathrm{~d}$.

Patsos, G., V. Hebbe-Viton., R. San Martin., C. Paraskeva., T. Gallagher, and A. Corfield 2005. Action of a library of 0 -glycosylation inhibitors on the growth of human colorectal cancer cancer cells in culture. Biochemical Society Transactions. 33: 721-723. doi: 10.1042/BST0330721.

Remaley, A.T., M. Ugorski., N. Wu., L. Litzky., S.R. Burger., J.S. Moore., M. Fukuda, and S.L. Spitalnik. 1991. Expression of human glycophorin $A$ in wild type and glycosylation-deficient Chinese hamster ovary cells. Role of $\mathrm{N}$ - and 0-linked glycosylation in cell surface expression. The Journal of Biological Chemistry. 266: 24176-83. PMID: 1748686.

Ricard-Blum, S. 2010. The collagen family. Cold Spring Harbor Perspectives in Biology. p. 1-19. Cold Spring Harb Perspect
Biol doi: 10.1101/cshperspect.a004978.

Rosenbloom, J, and D.J. Prockop. 1970. Incorporation of 3,4-Dehydroproline into Protocollagen and Collagen. The journal of Biological Chemistry. 245: 3381-3368. PMID: 5459639.

Schmidt, A., K. Datta, and A. Marcus. 1991. Peptidyl proline hydroxylation and the growth of a soybean cell culture. Plant Physiology. 96: 656-659. doi: 10.1104/pp.96.2.656

Seifert, G.J, and K. Roberts. 2007. The biology of Arabinogalactan proteins. Annual Review of Plant Biology. 58 137-161. doi: 10.1146/annurev.arplant. 58.032806.103801

Showalter, A.M. 1993. Structure and Function of Plant Cell Wall Proteins. Plant Cell. 59-23. doi: 10.1105/tpc.5.1.9.

Showalter, A.M. 2001. Arabinogalactan proteins: structure, expression and function. Cellular and Molecular Life Sciences. 58: 1399-1417. PMID: 11693522.

Showalter, A.M., B. Keppler., J. Lichtenberg., D. Gu, and L.R. Welch. 2010. A bioinformatics approach to the identification, classification, and analysis of hydroxyproline-rich glycoproteins. Plant Physiology. 153: 485-513. doi: 10.1104/pp.110.156554. Epub $2010 \mathrm{Apr}$ 15.

Shpak, E., E. Barbar., J.F. Leykam, and M.J. Kieliszewski. 2001. Contiguous hydroxyproline residues direct hydroxyproline arabinosylation in Nicotiana tabacum. The Journal of Biological Chemistry. 276: 1127211278. doi:10.1074/jbc.M011323200.

Shpak, E., J.F. Leykam, and M.J, Kieliszewski. 1999. Synthetic genes for glycoprotein design and the elucidation of hydroxyproline-0-glycosylation codes. Proceedings of the National Academy of Sciences 96: 14736-14741. PMCID: PMC2471.

Sola, R.J, and K. Griebenow. 2010. Glycosylation of therapeutic proteins: an effective strategy to optimize efficacy. BioDrugs. 24: 9-21. doi: 10.2165/11530550-000000000-00000.

Strasser, R. 2009. Localization of plant N-glycan processing enzymes along the secretory pathway. Plant Biosystems. 143: 636-642. doi: 10.1080/11263500903233391.

Strasser, R. 2013. Engineering of human-type $O$-glycosylation in Nicotiana benthamiana plants. Bioengineered. 4: 1-6. doi: 10.4161/bioe.22857. Epub 2012 Nov 12.

Takeuchi, T, and D.J. Prockop. 1969. Biosynthesis of abnormal collagens with amino acid analogues: I. Incorporation of l- azetidine-2-carboxylic acid and cis-4-fluoro-l-proline into protocollagen and collagen. Biochemica et Biophysica Acta. 176: 142-155. doi: 10.1016/0005-2795(69)90153-6.

Takeuchi, T., J. Rosenbloom, and D.J. Prockop. 1969. Biosynthesis of abnormal collagens with amino acid analogues: II. Inability of cartilage cells to extrude collagen polypeptides containing 1-azetidine-2-carboxylic acid or cis-4-fluoro-l-proline. Biochemica et Biophysica Acta. 175: 156-164. doi: 10.1016/0005-2795(69)90154-8.

Tan, L., F. Qiu., D.T.A. Lamport, and M.J. Kieliszewski. 2004. Structure of a hydroxyproline (Hyp)-arabinogalactan polysaccharide from repetitive Ala-Hyp expressed in 
transgenic Nicotiana tabacum. The Journal of Biological Chemistry. 279: 13156-13165. doi:10.1074/jbc.M311864200.

Tan, L., J.F. Leykam, and M.J. Kieliszewski. 2003. Glycosylation motifs that direct arabinogalactan addition to arabinogalactan-proteins. Plant Physiology. 132: 1362-1369. doi: 10.1104/pp.103.021766.

Tarp, M.A, and H. Clausen. 2008. Mucin-type $O$-glycosylation and its potential use in drug and vaccine development. Biochemica et Biophysica Acta. 1780: 546-563. doi: 10.1016/j.bbagen.2007.09.010.

Tian, E., G.T. Hagen Kelly., L. Shum., H.C. Hang., Y. Imbert., W.W. Young Jr., C.R. Bertozzi, and L.A. Tabak. 2004. An Inhibitor of O-Glycosylation Induces Apoptosis in NIH3T3 Cells and Developing Mouse Embryonic Mandibular Tissue. The Journal of Biological Chemistry. 48: 50382-50390. doi: 10.1074/jbc.M406397200.

Tsuboi, S, and M. Fukuda. 2001. Roles of O-linked oligosaccharides in immune responses. Bioessays. 1: 46-53.doi: 10.1002/1521-1878(200101)23:1<46::AID-B IES1006>3.0.CO;2-3.

Tullio, M.C.D., C. Paciolla., F.D. Vecchia., N. Rascio., S. D'Emerico., L.D. Gara., R. Liso, and O. Arrigoni. 1999. Changes in onion root development induced by the inhibition of peptidyl-prolyl hydro-xyllase and influence of the ascorbate system on cell division and elongation. Planta. 209: 424-434. PMID: 10550623.

van den Steen P., P. Rudd, M. Wormald, R. Dwek and G. Opdenakker. 2000. O-Linked glycosylation in focus. Trends Glycoscience Glycotechnology. 63: 35-49.

van den Steen, P., P.M. Rudd., R.A. Dwek, and G. Opdenakker. 1998. Concepts and principles of O-linked glycosylation. Critical Reviews in Biochemistry and Molecular Biology . 33: 151-208. PMID: 9673446.

Varki, A. 1993. Biological roles of oligosaccharides: all of the theories are correct. Glycobiology 3: 97-130. doi: 10.1093/glycob/3.2.97.

Velasquez, S.M., J.S. Salter., J. Gloazzo Dorosz., B.L. Petersen, and J.M. Estevez. 2012. Recent advances on the post-translational modifications of EXTs and their roles in cell expansion. Frontier Plant Physiology 3:1-6. doi: $10.3389 /$ fpls.2012.00093.

Velasquez, S.M., M.M. Ricardi., D.J. Gloazzo., P.V. Fernandez., A.D. Nadra., L. Pol-Fachin., J. Egelund., S. Gille., M. Ciancia., H. Verli., M. Pauly., A. Bacic., E.C. Olsen., P. Ulvskov., L.B. Petersen., C. Somerville., N.D. Iusem, and J.M. Estevez. 2011. Essential role of $O$-glycosylated plant cell wall extensins for polarized root hair growth. Science 332: 1401-1403. doi: 10.1126/science.1206657.

Vicré, M., C. Santaella., S. Blanchet., A. Gateau, and A. Driouich. 2005. Root border-like cells of Arabidopsis. Microscopical characterization and role in the interaction with rhizobacteria. Plant Physiology 138: 998-1008. doi: 10. 1104/pp.104. 051813.

Vlad, F., T. Spano., D. Vlad, F. Bou Daher., A. Ouelhadj, and P. Kalaitzis. 2007. Arabidopsis prolyl 4-hydroxylases are differentially expressed in response to hypoxia, anoxia and mechanical wounding. Plant Signaling \& Behavior 2:
368-369. doi: 10.1111/j.1399-3054.2007.00915.x.

Wilson Iain, B.H. 2002. Glycosylation of proteins in plants and invertebrates. Current Opinion in Structural Biology 12: 569-577. doi: 10.1016/S0959-440X (02) 00367-6.

Wopereis, S., D.J. Lefeber., E. Morava, and R.A. Wevers. 2006. Mechanisms in protein $O$-glycan biosynthesis and clinical and molecular aspects of protein $O$-glycan biosynthesis defects: a review Clinical Chemistry 52: 574-600. doi: 10.1373/clinchem.2005.063040.

Xu C., T. Takáč., C. Burbach., D. Menzel, and J. Šamaj. 2011. Developmental localization and the role of hydroxyproline rich glycoproteins during somatic embryogenesis of banana (Musa spp. AAA). BMC Plant Biology 11: 38. doi: 10.1186/1471-2229-11-38.

Xu, J., L. Tan., D.T.A. Lamport., A.M. Showalter, and M.J. Kieliszewski. 2008. The 0-Hyp glycosylation code in tobacco and Arabidopsis and a proposed role of Hyp-glycans in secretion. Phytochemistry 69: 1631-1640. doi: 10.1016/j.phytochem.2008.02.006. Epub 2008 Mar 25.

Zhang, X., Y. Ren, and J. Zhao. 2008. Roles of extensins in cotyledon primordium formation and shoot apical meristem activity in Nicotiana tabacum. Journal of Experimental Botany 59: 4045-4058. doi: $10.1093 / \mathrm{jxb} / \mathrm{ern} 245$. 